\title{
La position de l'Agence du revenu du Canada dans la technique du pipeline en planification post mortem
}

\author{
Éric Bélanger ${ }^{1}, \mathrm{CPA}, \mathrm{CA}, \mathrm{MBA}, \mathrm{M}$. Fisc. \\ Université du Québec à Chicoutimi
}

\section{INTRODUCTION}

\section{La mort n'est jamais un évènement heureux. Comme si ce n'était pas suffisant, dans ces jours de deuil, nous nous retrouvons confrontés à un autre concept peu populaire, celui de l'impôt.}

La mort n'est jamais un évènement heureux. Comme si ce n'était pas suffisant, dans ces jours de deuil, nous nous retrouvons confrontés à un autre concept peu populaire, celui de l'impôt. Bien que la période qui suit ces tristes évènements ne soit pas la plus appropriée pour discuter de fiscalité, une bonne planification fiscale demeure essentielle afin d'optimiser la situation du défunt et de sa succession.

\section{EXEMPLE DE DOUBLE IMPOSITION}

Afin de bien saisir une situation de double imposition au moment du décès d'un actionnaire d'une société privée, nous présentons un exemple. M. Malenpoint détient les actions d'une société de gestion (GESCO). Celles-ci ont une juste valeur marchande de $800000 \$$, un prix de base rajusté de 10000 \$ ainsi qu'un capital versé de 10000 \$. En vertu de la Loi de l'impôt sur le revenu, l'action-
Pour ces motifs, il s'est développé différentes techniques de planification fiscale au fil des années. Le présent ouvrage abordera l'une de ces planifications, soit la technique du pipeline. Celle-ci ne fut pas toujours la solution retenue, mais dans le contexte actuel de la fiscalité, elle est souvent priorisée.

L'objectif sera de démontrer les problématiques fiscales au décès d'un actionnaire d'une société privée. Nous démontrerons que la technique du pipeline permet de régler ce problème. Nous expliquerons cette technique tout en exposant la position de l'Agence du revenu du Canada (ARC).

naire sera présumé avoir disposé de la totalité de ses biens immédiatement avant son décès ${ }^{2}$ lui occasionnant un gain en capital de 790000 \$. Lorsque les héritiers se verront attribuer personnellement les biens de GESCO, ils payeront un dividende de liquidation $^{3}$. Ce dividende présumé causera une double imposition. Voici les conséquences fiscales présentées dans le tableau ${ }^{4}$ :

\begin{tabular}{|llr|}
\hline \multicolumn{3}{|c|}{ M. MALENPOINT } \\
\hline Gain en capital imposable & Produit de disposition & 800000 \\
Impôt : $197382 \$$ (A) & Moins : Prix de base rajusté & $\frac{(10000)}{790000}$ \\
(taux combiné de 49,97\%) & Gain en capital & $50 \%$ \\
& Taux d'inclusion & 395000 \\
\hline & Gain en capital imposable & \\
\hline Dividende réputé & LA SUCCESSION & 800000 \\
Impôt : 304 466 \$ & Argent reçu & $(10000)$ \\
(taux combiné de 38,54 \%) & Moins : capital versé & 790000 \\
\hline Impact fiscal total $(\mathbf{A})+(\mathbf{B}): 501848 \$$ & Dividende réputé & \\
\hline
\end{tabular}


La situation de double imposition surviendra lorsque la succession ne sera pas en mesure de vendre les actions à un tiers.

La situation de double imposition surviendra lorsque la succession ne sera pas en mesure de vendre les actions à un tiers. Effectivement, la succession obtient les actions avec un PBR égal à la $\mathrm{JVM}^{5}$. Lors de la vente à un tiers, le gain en capital sera nul et aucun impôt ne sera payable. Dans le cas d'une société de placements, aucun acheteur ne voudra acheter de telles actions. Le risque de double imposition est transféré à la succession et il sera toujours présent dans le cas d'une société de gestion ${ }^{6}$.
Nous constatons que sur une JVM de 800000 \$, le gouvernement aura récolté 501848 \$ en impôt, soit une imposition de l'ordre de $62,7 \%$. Ce pourcentage ne tient pas compte de l'impôt latent sur les biens de GESCO et présume que le choix du paragraphe 164(6) L.I.R. n'est pas effectué. Il devient donc évident qu'une forme de planification sera nécessaire afin de contrer l'effet de la double imposition. Comme le mentionnait Louis Jeannotte : « Quelle collectivité aspire à imposer deux fois la même richesse ou le même revenu? » ${ }^{7}$. Il existe essentiellement deux façons d'éviter une double imposition. Il y a l'utilisation du paragraphe 164(6) L.I.R. et la technique du pipeline. Dans le cadre du présent ouvrage, nous nous concentrons uniquement sur la technique du pipeline.

\section{CHOIX : ÉLIMINATION DU GAIN EN CAPITAL OU DU DIVIDENDE}

Le tableau suivant illustre l'évolution des taux d'imposition de 2012 à 2014.

\begin{tabular}{|c|c|c|c|}
\hline \multicolumn{4}{|c|}{ TAUX D'IMPOSITION MARGINAUX COMBINÉS POUR LES PARTICULIERS AU } \\
QUÉBEC & $\begin{array}{c}\text { Dividendes non } \\
\text { déterminés }\end{array}$ \\
\hline Année & Gain en capital & Dividendes déterminés & $36,35 \%$ \\
2012 & $24,11 \%$ & $32,81 \%$ & $38,54 \%$ \\
2013 & $24,99 \%$ & $35,22 \%$ & $39,78 \%$ \\
\hline 214 & $24,99 \%$ & $35,22 \%$ & $\%$ \\
\hline
\end{tabular}

Il ressort que le gain en capital est plus avantageux que le dividende au Québec actuellement. Cependant, ce ne fut pas toujours le cas. Il y a quelques années, le dividende était le plus attrayant. De plus, la situation n'est pas nécessairement la même dans les autres provinces canadiennes.

\section{LA TECHNIQUE DU PIPELINE}

La présente section a pour objectif de décrire, étape par étape, une planification de type pipeline. Au moment du décès de M. Malenpoint, la succession constitue une nouvelle société de gestion (ci-après « NOUCO ») et souscrit à cent actions ordinaires de son capital-actions pour un montant de cent dollars. Ensuite, la succession vend les actions qu'elle détient dans le capitalactions de GESCO en faveur de NOUCO qui lui émet en contrepartie un billet à demande d'un montant équivalent à la JVM des actions vendues,
Des deux techniques de planification post mortem qui évitent la double imposition, celle du paragraphe 164(6) L.I.R. permet d'éviter le gain en capital et celle du pipeline élimine le dividende présumé. Compte tenu que le gain en capital est actuellement imposé à des taux moindres que le dividende, l'analyse de la technique du pipeline prend tout son sens.

soit 800000 \$. Par la suite, il faudra soit fusionner ${ }^{8}$ GESCO et NOUCO, soit liquider ${ }^{9}$ GESCO dans NOUCO. Dans les deux cas, aucun impôt ne sera payable ${ }^{10}$. L'opération suivante consiste à rembourser le billet à payer à la succession. Ce remboursement peut s'effectuer en argent comptant ou à même les biens de NOUCO. Cette dernière option amène alors une disposition imposable pour NOUCO. Finalement, nous pouvons procéder à la dissolution de NOUCO. Cette étape n'étant pas obligatoire, 
nous pouvons aussi continuer à utiliser NOUCO afin d'effectuer des placements selon les besoins de chaque cas. Cette planification aura permis de conserver seulement le gain en capital de
M. Malenpoint et d'éviter le dividende présumé pour la succession. Le tableau suivant présente le sommaire des impacts fiscaux suite à cette planification $^{11}$ :

\begin{tabular}{|c|c|c|}
\hline \multicolumn{3}{|c|}{ M. MALENPOINT } \\
\hline Gain en capital imposable & Produit de disposition & 800000 \\
\hline Impôt : 197382 \$ (A) & Moins : Prix de base rajusté & $(10000)$ \\
\hline (taux combiné de 49,97\%) & Gain en capital & 790000 \\
\hline & Taux d'inclusion & $50 \%$ \\
\hline & Gain en capital imposable & 395000 \\
\hline \multicolumn{3}{|c|}{ LA SUCCESSION } \\
\hline \multicolumn{3}{|c|}{$\begin{array}{l}\text { Aucun dividende réputé, car les biens ont été distribués à la succession par le remboursement du } \\
\text { billet à payer. }\end{array}$} \\
\hline Impact fiscal total (A) : 197382 \$ & & \\
\hline
\end{tabular}

$\mathrm{Au}$ net, cette planification permet une économie d'impôt de l'ordre de 304466 \$, soit le montant du dividende présumé de la succession sans la technique du pipeline.

\section{POSITION ADMINISTRATIVE DE L'ARC QUANT AU PIPELINE}

L'ARC a eu l'occasion de se prononcer sur la technique du pipeline dans le cadre de plusieurs interprétations techniques. Elle n'est pas nécessairement contre cette planification, mais elle fixe plusieurs exigences. Cette section a pour objectif de les examiner plus en détail.

L'ARC est à la recherche de plusieurs critères afin d'accepter le pipeline. Le non-respect de l'un d'eux amène l'ARC à attaquer la transaction et à émettre une cotisation. De façon succincte, voici les éléments recherchés :

- GESCO doit demeurer une entité juridique distincte (i.e. que cette société n'est pas liquidée dans une autre société ou fusionnée avec une autre société) pour une période d'au moins une année ${ }^{12}$.

- Durant cette période d'une année, GESCO doit maintenir ses activités. Il faut donc qu'elle continue à exploiter son entreprise de la même façon qu'elle le faisait auparavant ${ }^{13}$.

- Sans être une exigence, le fait de continuer à utiliser NOUCO pour détenir, gérer et transiger divers placements suite à la planification ainsi que le remboursement progressif du billet aident à l'obtention d'une décision favorable. ${ }^{14}$

- Dans le cas où les liquidités constituent le seul actif d'une société de gestion et qu'elles sont distribuées aux actionnaires sans le paiement d'un dividende, l'ARC a indiqué qu'elle attaquerait cette planification en vertu du paragraphe 84(2) L.I.R. ${ }^{15}$. Cette position est confirmée dans plusieurs interprétations techniques ${ }^{16}$.

Une constante ressort de l'analyse de l'ensemble des positions administratives de l'ARC. Lorsqu'elle se prononce sur une planification du type pipeline, elle donne toujours son opinion sur l'application de l'article $84.1 \quad$ L.I.R., du paragraphe $84(2)$ L.I.R. ainsi que du paragraphe 245(2) L.I.R.

L'ARC stipule que l'exigence de l'entité juridique distincte ainsi que le maintien des activités pendant une période d'une année faisaient partie des opérations projetées d'interprétations techniques précédentes ${ }^{17}$ et qu'il ne s'agit pas d'une obligation. Toutefois, celle-ci admet qu'elles ont contribué à l'acceptation de la transaction pipeline.

Dans les interprétations techniques les plus récentes émises par l'ARC ${ }^{18}$, le délai exigé est hachuré dans le texte, ce qui rend impossible la lecture du délai exigé. Il n'est plus indiqué que la période d'attente doit être d'au moins une année. Cette évolution de la position administrative porte à croire qu'un délai plus court est accepté, bien qu'en doutent certains auteurs ${ }^{19}$. 
Il est important de comprendre qu'il n'y a aucune jurisprudence traitant spécifiquement de la technique du pipeline. Jusqu'à présent, les planifi-

\section{CONCLUSION}

\section{À titre de conseillers fiscaux, il est de notre devoir de recommander la planification la plus avantageuse pour nos clients. Le pipeline n'est pas nécessairement la technique à utiliser dans toutes les situations, mais nous devons l'employer lorsque c'est opportun.}

Rappelons que l'objectif de cet article consiste à comprendre l'utilité de la technique du pipeline et d'exposer la position de l'ARC. Néanmoins, un problème provient du fait que les autorités fiscales ont une position qui n'est pas en accord avec celle des praticiens du milieu. De plus, il n'existe aucune cause ayant été portée devant les tribunaux qui traite spécifiquement de cette planification. Par conséquent, deux choix s'offrent aux contribuables : suivre les directives fournies par les autorités fiscales ou persister et courir le risque de devoir défendre leur position devant les tribunaux.

À titre de conseillers fiscaux, il est de notre devoir de recommander la planification la plus avantageuse pour nos clients. Le pipeline n'est pas nécessairement la technique à utiliser dans toutes les situations, mais nous devons l'employer lorsque c'est opportun. Toutefois, la position de l'ARC est claire. Si nous ne respectons pas ses exigences, la transaction sera automatiquement cotisée. Il s'agit bien sûr du genre d'information que nous devons communiquer au client. Pour certains d'entre eux, le risque de débattre de l'affaire devant les tribunaux est suffisant pour ne pas aller de l'avant avec un pipeline classique.

Pour toutes ces raisons, ce sujet a fait couler beaucoup d'encre et ne semble pas avoir fini de le faire. Tant et aussi longtemps qu'un contribuable n'aura pas le courage de confronter le gouvernement, nous risquons fort possiblement de continuer à mettre en place des planifications adaptées à la position de l'ARC qui n'optimisent pas totalement la situation de notre client. cateurs fiscaux préfèrent se plier aux exigences de l'ARC.

\section{BIBLIOGRAPHIE ET NOTES}

${ }^{1}$ Éric Bélanger est comptable et fiscaliste. Il travaille comme professeur de fiscalité à l'Université du Québec à Chicoutimi.

${ }^{2}$ Par. 70(5) L.I.R.

${ }^{3}$ Par. 88(2) L.I.R.

${ }^{4}$ Puglièse, Claudine, « Planification post mortem et successorale - Mise à jour » dans Congrès 2007, Montréal, Association de planification fiscale et financière, 2007.

${ }^{5}$ Supra note 2.

${ }^{6}$ Jeannotte, Louis, « Planification fiscale post mortem : Comment éviter la double imposition » dans Congrès 2003, Montréal, Association de planification fiscale et financière, 2003.

${ }^{7}$ Supra note 6.

${ }^{8}$ Art. 87 L.I.R.

${ }^{9}$ Art. 88 L.I.R.

${ }^{10}$ Art. 87 L.I.R. et art. 88 L.I.R.

${ }^{11}$ Supra note 4.

${ }^{12} \mathrm{ARC}$, Interprétation technique 2006-0170641E5, « Distribution of Corporate Property » (29 juin 2006) (CCH, Tax Window Files).

${ }^{13}$ Interprétation technique 2006-0170641E5, supra note 12.

${ }^{14} \mathrm{ARC}$, Interprétation technique 2002-0154223, « Post Mortem Planning » (13 novembre 2002) (CCH, Tax Window Files).

${ }^{15}$ Moraitis, Nick et Manu Kakkar, « Planification postmortem : Du nouveau concernant l'opération pipeline » (avril 2011) 11:2 Actualités fiscales pour les propriétaires exploitants.

${ }^{16}$ ARC, Interprétation technique 2010-0389551R3, « Post-Mortem Planning - Withdrawal of Ruling » (12 novembre 2010) (CCH, Tax Window Files); ARC, Interprétation technique 2009-0326961C6, « Distribution of Corporate Property » (9 octobre 2009) (CCH, Tax Window Files).

${ }^{17}$ ARC, Interprétation technique 2005-0142111R3, « Post Mortem Planning » (2 novembre 2005) (CCH, Tax Window Files); ARC, Interprétation technique 20020154223, « Post Mortem Planning 》 (13 novembre 2002) (CCH, Tax Window Files).

${ }^{18}$ ARC, Interprétation technique 2010-0388591R3, « Post Mortem Planning » (4 avril 2011) (CCH, Tax Window Files); ARC, Interprétation technique 20100377601R3, « Post Mortem Planning » (11 novembre 2010) (CCH, Tax Window Files).

${ }^{19}$ Voir notamment Yager, Jim, " Planification post mortem d'opérations pipeline » (mai 2011) 19:5 Faits saillants en fiscalité canadienne. 